\title{
Tissue-specific and exosomal miRNAs in lung cancer radiotherapy: from regulatory mechanisms to clinical implications
}

This article was published in the following Dove Press journal: Cancer Management and Research

\author{
Long Long',* \\ Xue Zhang ${ }^{1} *$ \\ Jian $\mathrm{Bai}^{2,3}$ \\ Yizhou $\mathrm{Li}^{4}$ \\ Xiaolong Wang ${ }^{5}$ \\ Yunfeng Zhou' \\ 'Department of Radiation and Medical \\ Oncology, Zhongnan Hospital of Wuhan \\ University, Wuhan 43007I, People's \\ Republic of China; ${ }^{2}$ Department of \\ Oncology, Zhongnan Hospital of Wuhan \\ University, Wuhan, People's Republic of \\ China; ${ }^{3}$ Hubei Key Laboratory of Tumor \\ Biological Behaviors and Hubei Cancer \\ Clinical Study Center, Wuhan, 43007I, \\ People's Republic of China; ${ }^{4}$ Department \\ of Orthopaedics, Zhongnan Hospital of \\ Wuhan University, Wuhan 43007I, \\ People's Republic of China; ${ }^{5}$ Department \\ of Urology, Research Lab/LIFE-Zentrum, \\ University of Munich (LMU), München, \\ Germany
}

*These authors contributed equally to this work
Correspondence: Yunfeng Zhou Department of Radiation and Medical Oncology, Zhongnan Hospital of Wuhan University, 169 East Lake Road, Wuchan, Wuhan 43007I, People's Republic of China

Tel +86 2767813162

Fax +8602767813162

Email yfzhouwhu@163.com

\begin{abstract}
Lung cancer is the most prevalent and deadly malignancy. Radiotherapy is a major treatment modality for lung cancer. Nevertheless, radioresistance poses a daunting challenge that largely limits the efficacy of radiotherapy. There is a pressing need for deciphering molecular mechanisms underlying radioresistance and elucidating novel therapeutic targets for individualized radiotherapy. MicroRNAs are categorized as small noncoding RNAs that modulate target-gene expression posttranscriptionally and are implicated in carcinogenesis and cancer resistance to treatment. Overwhelming evidence has unraveled that tissue-specific miRNAs are essential for regulation of the radiosensitivity in lung cancer cells through a complex interaction with multiple biological processes and radiation-induced pathways. Moreover, exosome-derived miRNAs are a novel horizon in lung cancer treatment in which exosomal miRNAs act as potential diagnostic and therapeutic biomarkers of radiotherapy. In the present review, we discuss the mediation of key biological processes and signaling pathways by tissue-specific miRNAs in lung cancer radiotherapy. Additionally, we provide new insight into the potential significance of exosomal miRNAs in radiation response. Lastly, we highlight miRNAs as promising predictors and therapeutic targets to tailor personalized lung cancer radiotherapy.
\end{abstract}

Keywords: lung cancer, microRNAs, exosome, radioresistance, personalized radiotherapy

\section{Introduction}

Lung cancer is a cardinal cause of fatal malignancy, with 234,030 new cases and 154,050 deaths estimated to have occurred in the US in $2018 .{ }^{1}$ It can be subdivided into two types: small-cell lung cancer (SCLC) and non-SCLC (NSCLC). Clinically, NSCLC is the most frequent subtype, making up $85 \%$ of diagnosed cases. ${ }^{2}$ Radiotherapy (RT) is a major treatment modality and sometimes curative in lung cancer patients. ${ }^{3}$ Nevertheless, radioresistance poses a daunting impediment, which largely undermines the efficacy of RT. ${ }^{4}$ The 5 -year overall survival of lung cancer remains poor (18\%), owing to local recurrence and distant metastasis. ${ }^{1,5}$ Therefore, it is imperative to decipher key mechanisms underlying radioresistance and identify novel therapeutic targets for individualized RT.

miRNAs, an abundant family of short (19-25 nucleotides) noncoding RNAs, can negatively modulate gene expression upon binding to target mRNAs. Aberrant expression of miRNAs can regulate diverse cellular processes, including cell development, migration, and apoptosis. ${ }^{6}$ In recent years, accumulating evidence has revealed that miRNAs can influence radiation response remarkably (Figure 1). ${ }^{7}$ 


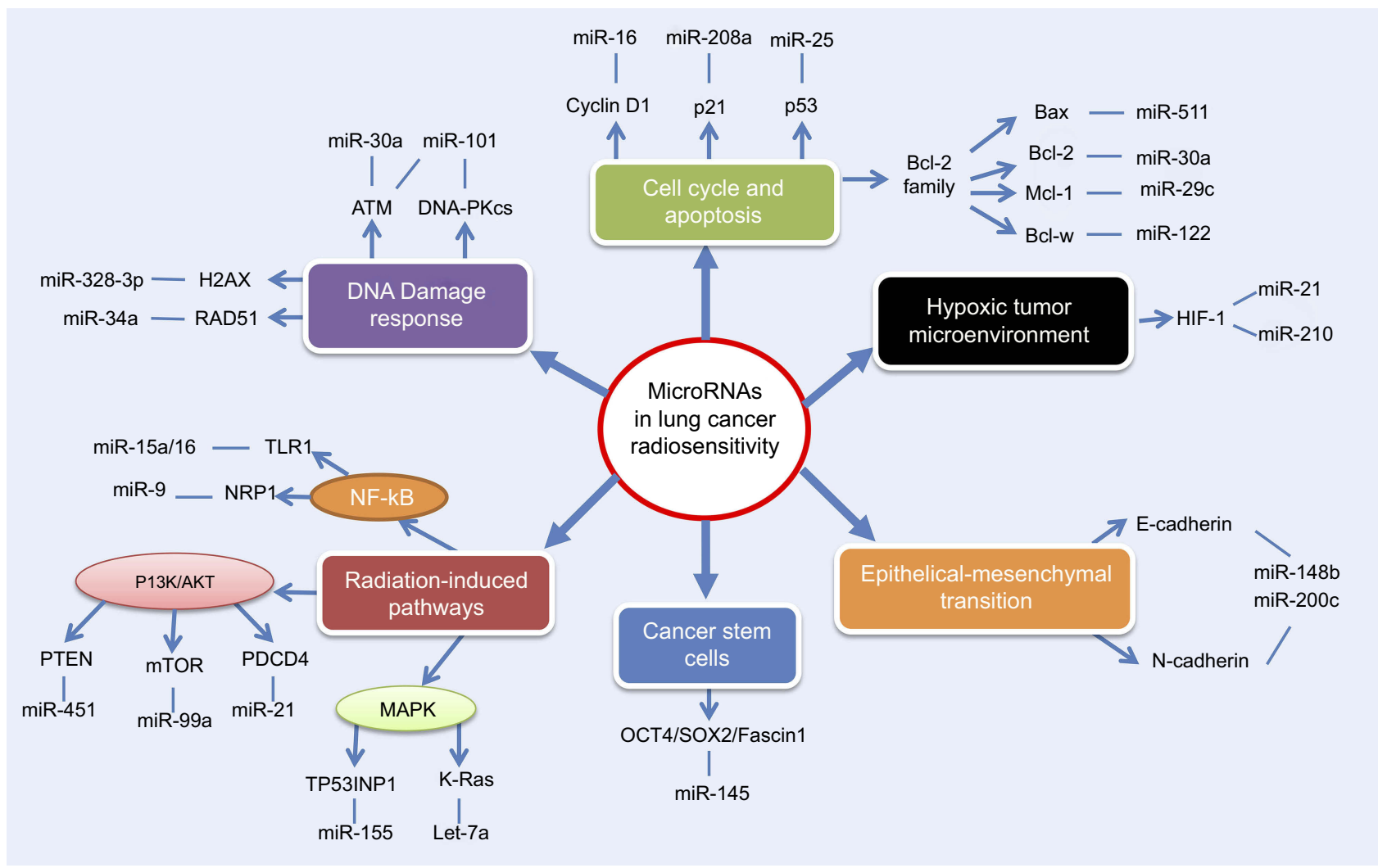

Figure I An overview of tissue-specific miRNAs in the regulation of lung cancer radiosensitivity.

Notes: MiRNAs exert essential function to regulate the radiosensitivity of lung cancer cells, through complex interaction with multiple biological processes including DNA damage response, cell cycle and apoptosis, hypoxic tumor microenvironment, epithelial-mesenchymal transition, cancer stem cells and radiation-induced signaling pathways.

Additionally, miRNA profiling in tumor tissue or circulating body fluid is recognized to correlate with radiosensitivity, holding considerable promise to predict clinical response. ${ }^{8}$

Of note, exosome-derived miRNAs have offered an amazing outlook in radiation research. ${ }^{9}$ Exosomes are small membrane-derived vesicles $(50-150 \mathrm{~nm})$ released by multiple cell types, including cancer cells. Exosomes convey different cargoes containing miRNAs, mRNAs, and proteins specializing in intercellular communication. ${ }^{10}$ It is increasingly evident that exosomal miRNA profiles can be altered in radiation response. ${ }^{9}$ Radiation-related miRNAs are possibly transported by exosomes, influencing the proliferation and radiosensitivity of lung cancer cells. ${ }^{11}$

In this work, we discuss the modulation of key biological processes and signaling pathways by tissue-specific miRNAs in lung cancer RT. Furthermore, we present a new insight into the significance of exosomal miRNAs in radiation response. Finally, we emphasize miRNAs as promising predictors and therapeutic targets to tailor personalized RT.

\section{Regulatory roles of tissue-specific miRNAs in lung cancer radiosensitivity DNA-damage response}

RT utilizes ionizing radiation (IR) to generate free radicals and intermediate ions, which damage tumor cells at different levels, especially cellular DNA. It results in DNA single-strand breaks or double-strand breaks (DSBs), initiating diverse signaling networks to repair. ${ }^{12}$ DNA- damage response (DDR) is a pivotal biological process affecting radiosensitivity, in which DSB repairs are the most widespread events, containing homologous recombination (HR) and nonhomologous end joining. ${ }^{12}$ Numerous molecules exert remarkable effects during DDR, including sensors (eg, H2AX), signal transducers (eg, ATM), and effectors (eg, the DNA-dependent PK catalytic subunits [PKcs], RAD51 and BRCA1/BRCA2). ${ }^{13}$

Several well-established miRNAs interfere with IRinduced DNA-damage sensing or repair, via complex interplay with DDR components (Figure 2). miR328-3p can 


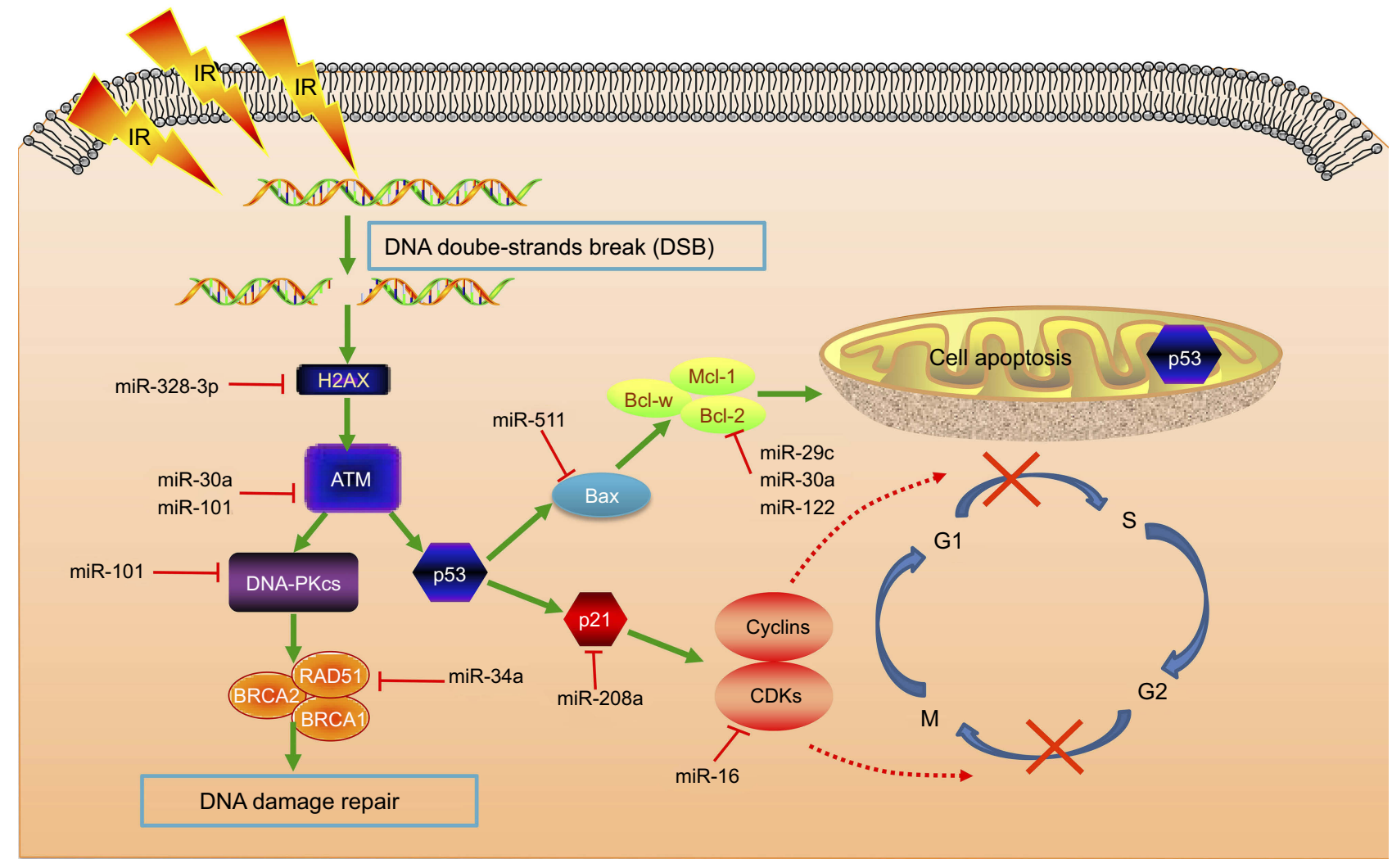

Figure 2 miRNAs in DNA-damage response, cell cycle, and apoptosis.

Notes: Radiotherapy utilizes ionizing radiation to generate free radicals and intermediate ions, which damage tumor cells at different levels, especially with DNA double-strand breaks, initiating diverse signaling pathways to repair. Cyclins and $C D K s$ are indispensable regulators of cell-cycle transition, which can be suppressed by checkpoints at $G_{1} / S$ and $G_{2} / M$ interphases, ultimately to arrest cell-cycle progression and allow enough time for DNA-damage repair. If DNA damage is too severe to repair, cells will proceed to apoptosis or programmed cell death.

Abbreviations: IR, ionizing radiation; PKcs, PK catalytic subunits.

augment DSBs through upregulating H2AX, conducive to radiosensitization. ${ }^{14}$ ATM is a determining factor in and prime responder to DSBs, triggering IR-induced cellular events after phosphorylation. ATF1, a mediator of phosphorylation in the ATM pathway, serves as a direct target of miR30a. It has been revealed that miR30a enhances radiosensitivity through reducing ATF1 activity and thus diminishing ATM phosphorylation. ${ }^{15}$ Ectopic miR101 expression efficiently attenuates ATM and DNA-PKcs to repress DDR, radiosensitizing cells with much higher endogenous miR101. ${ }^{16}$ Preclinical data has suggested that miR1323 and accumulation of DNA-PKcs are concomitantly increased after radiation. Conversely, knockout of miR1323 is unable to recruit DNA-PKcs in DDR. ${ }^{17}$ Moreover, RAD51 acts as a critical player in HR, catalyzing new DNA transfer and recombination within damaged areas. miR34a overexpression can negatively regulate HR by posttranscriptionally suppressing RAD51. ${ }^{18}$ Thoroughly elucidating the regulation of miRNAs in DDR will probably dig deeply into IRinduced biological processes for overcoming radioresistance.

\section{Cell cycle and apoptosis}

As we know, the cell cycle contains four phases: $G_{1}, S, G_{2}$, and $M$. Cells in the $\mathrm{G}_{2} / \mathrm{M}$ phase possess the highest sensitivity to IR, whereas radiosensitivity reduces from the $G_{1}$ to S phase. ${ }^{19}$ Cyclins and CDKs are indispensable regulators in cell-cycle transition from one phase to another. In radiationinduced stress, cyclins and CDKs can be inhibited by the checkpoints at the $G_{1} / S$ and $G_{2} / M$ interphases to arrest cell progression and allow enough time for DDR (Figure 2). ${ }^{19}$ The miR16 family directly represses cyclin D1, preventing cells from proceeding into the $\mathrm{S}$ phase. Simultaneously, numerous cell-cycle genes, such as CCND3, CCNE1, and CDK6, are silenced by miR16, collectively triggering $G_{1}$ arrest. ${ }^{20}$ Moreover, miR30a-expressing A549 cells specifically block $\mathrm{G}_{2} / \mathrm{M}$ arrest, accompanied by inhibiting transcription factors p53 and $21 .{ }^{15}$

If IR-induced DNA damage is too severe to repair, cells will proceed to apoptosis or programmed cell death (Figure 2). ${ }^{7}$ miRNAs modulate expression of the proapoptotic factors p53, Bax, caspase 3 and antiapoptotic factors 
$\mathrm{Bcl} 2$ and $\mathrm{Mcl1} .^{21} \mathrm{p} 21$ is a p53-inducible factor, which concomitantly abrogates the PCNA and cyclinD1-CDK4/ 6 compounds, controlling cell-cycle progression. ${ }^{11}$ Induced by radiation, miR208a overexpression amplifies cell survival and obstructs cycle distribution, with p21 as the direct target. Nevertheless, depletion of miR208a possesses inverse radiosensitizing effects. ${ }^{11}$ miR25 overexpression can protect H226 NSCLC cells from IR-induced apoptosis by impairing BTG2, an antiproliferation factor in the p53 apoptotic pathway. ${ }^{22}$ Also, $B C L 2$-family genes (eg, BCL2, MCL1, BCL2L2, and $B A X$ ) are critical in the intrinsic mitochondrial pathway, conducive to apoptosis as well. ${ }^{23}$ Reestablishment of miR29c significantly dampens $\mathrm{Bcl} 2$ and Mcl1, thus activating cell apoptosis. ${ }^{24}$ miR122 exerts radiosensitizing effects by reducing Bcl212, another pro-survival modulator. ${ }^{25} \mathrm{Bax}$ is an apoptosis activator in the Bcl2 family and p53 pathway. miR30a upregulation attenuates Bcl2 and Bax protein after irradiation, amplifying apoptotic rates. ${ }^{15}$ Also, miR511 can enhance Bax to block the growth of RT-resistant A549 cells. ${ }^{26}$ Knockdown of miR95 speeds up IR-induced apoptosis, with an increase in caspase 3/9 and reduction in Bcl2. ${ }^{27}$ Also, there exist other mechanisms in miRNA-mediated apoptosis. miR124 decreases cell survival via inhibiting STAT3, and miR198 exerts a similar effect by repressing the HGFcMet pathway. ${ }^{28,29}$

\section{Hypoxic tumor microenvironment}

The hypoxic microenvironment is a hot spot in cancer research that strikingly facilitates tumor development, invasion, and metastasis. ${ }^{30}$ It has been shown that HIF1, a pivotal transcription factor, can help tumor cells adapt to hypoxic conditions. ${ }^{31}$ The precise roles of HIF1 in radiosensitivity are highly complex: RT can speed up the activation of HIF1, and HIF1 conversely influences radiation response through multiple signaling pathways. ${ }^{32}$ HIF1 upregulation triggers tumor angiogenesis, enhances IR-induced reoxygenation, and lessens glucose metabolism, collectively reducing RT efficacy. ${ }^{33}$ In RT-resistant NSCLC cells, HIF1 $\alpha$ is elevated by miR21, with an increase of key enzymes in glycolysis, whereas, blocking HIF1 $\alpha$ impedes glycolysis and enhances cell radiosensitization. ${ }^{34}$ Stable miR210 expression is implicated in the stabilization of HIF and confers more glycolytic properties, while inhibiting HIF1 effectively attenuates radioresistance, confirming the strong link between miR210 and HIF $1 .^{35}$ Therefore, the intricate regulatory interfaces between miRNA and the hypoxic tumor microenvironment may provide new insight into radioresistance.

\section{Epithelial-mesenchymal transition}

Epithelial-mesenchymal transition (EMT) is a phenotypic transition to enable epithelial tumor cells to acquire fibroblastoid-like morphology, contributing toward tumor invasion and resistance to treatment. ${ }^{36}$ It has been shown that miR124 can target PRRX1 in colorectal cancer cells and induce EMT after radiation. ${ }^{37}$ Furthermore, miR148b upregulation has been reported to suppress EMT by increasing E-cadherin and decreasing N-cadherin and vimentin. Contrastingly, downregulation of miR $148 \mathrm{~b}$ inhibits E-cadherin but elevates $\mathrm{N}$-cadherin and vimentin. ${ }^{38}$ In a panel of different cancer cells, miR200c mitigated EMTrelated processes, including vascular formation, invasion, and migration, through complex signaling networks. In response to radiation, miR200c remarkably repressed E-cadherin and EphA2 and enhanced N-cadherin in A549 NSCLC and U251 glioblastoma cells. ${ }^{39}$ Nevertheless, research on how miRNAs mediate RT-related EMT in lung cancer is still largely in its infancy, and more studies are needed to discover more about the mechanisms therein.

\section{Cancer stem cells}

Cancer stem cells (CSCs) possess high potential for selfrenewal and differentiation, and possibly display inherent resistance to treatment, including $\mathrm{RT}^{40}$ Despite limited investigations in recent years, miRNAs have shown tantalizing promise in modulating lung cancer radioresistance via CSCs. In lung adenocarcinoma, miR145 suppresses the proliferation of CSCs and improves radioresistance by inhibiting the transcription factors OCT4, SOX2, and fascin 1, which are crucial for maintaining cancer stemness. ${ }^{41}$ Moreover, miR21 and miR95 arere remarkably upregulated in theALDH $1^{+} \mathrm{CD} 133^{+}$subpopulation of lung CSCs, conferring a radioresistant phenotype. ${ }^{42}$ Another observation implied that miR18a-5p promotes the radiosensitivity of $\mathrm{CD}_{133^{+}}$stem-like cells through reduction of ATM and HIF $1 \alpha$ at both mRNA and protein levels. ${ }^{43}$ The regulation of miRNAs in CSCs remains an attractive research direction for lung cancer radioresistance, and needs to be investigated thoroughly for innovative therapies.

\section{Radioinduced signal-transduction pathways}

Some well-delineated signaling pathways might be paramount in the modulation of radiosensitivity. For instance, PI3K/Akt, NFkB, and MAPK are viewed as important survival pathways in radiation response. ${ }^{44}$ miRNAs can exert effective function in modulation of the radioinduced 
signaling pathways. Evidence suggests that miR373 and miR126 promote IR-induced apoptosis through the PI3KAkt pathway in lung cancer. ${ }^{45,46}$ PTEN is an inhibitory modulator of Akt, and acts as a direct target of miR451. miR451 overexpression has been reported to activate PTEN posttranscriptionally after irradiation, amplify suppressive impact, and facilitate A549-cell radiosensitization. ${ }^{47}$ As a key downstream component of the PI3K-Akt pathway, mTOR is directly mediated by miR99a. miR99a expression is significantly upregulated in RT-sensitive cells, while mTOR overexpression inversely diminishes cell apoptosis and exacerbates radioresistance. ${ }^{48}$ miR21 triggers activation of PI3K-Akt-mTOR signal cascades via deregulating PDCD4. In addition, miR21 can mediate bystander effects in unirradiated cells, through transformation of the TGF $\beta_{1}$ pathway. ${ }^{49}$ Strikingly, there exist intricate intersections among different signaling pathways. For example, miR9 may simultaneously block VEGFR2-PI3K-NFkB signaling. ${ }^{50}$ miR9 and let7 are able to target the NFkB1 transcript and blunt the antiapoptotic impacts of $\mathrm{NF \kappa B}$ signaling. ${ }^{51}$ Moreover, miR15a/16 exhibits similar effects via direct modulation of TLR1-NFkB pathways. ${ }^{52}$

Analogous to the PI3K-Akt pathway, the MAPK pathway has been reported to be mediated by miRNAs. The Ras protein can transmit downstream signals of the MAPK pathway. Let7a overexpression represses Kras, a member of Ras family, leading to radiosensitivity of lung cancer cells. ${ }^{53}$ Additionally, miR155 stimulated downstream of p53 and p38-MAPK pathways, functionally modulating IR-induced senescence, partly via TP53INP1. ${ }^{54}$ miR214 deregulation can effectively alter p38-MAPK and PI3K-signaling networks. ${ }^{55}$ The engagement of miRNAs in proverbial oncogenic pathways emphasizes the necessity to exploit biological mechanisms of radioresistance (Table 1, Figure 1).

\section{Circulating miRNAs and exosomal miRNAs in lung cancer radiotherapy Circulating miRNAs}

Apart from tissue-specific miRNAs, more oncologists have shifted their focus into circulating miRNAs, which are present in such body fluids as serum, saliva, urine, and bronchoalveolar lavage. ${ }^{56}$ miRNAs can be released from parental cells under different conditions, such as apoptosis, injury, or inflammation. During the process, microvesicle secretion (eg, exosomes and shedding vesicles), can free membrane-encapsulated cellular fragments containing miRNAs from donor cells. ${ }^{57}$ It has been identified in functional analysis that circulating miRNAs may evade degradation, probably owing to the internalization of phospholipid membrane-enclosed microvesicles or the presence of RNA-binding protein complexes. ${ }^{58}$

\section{Circulating miRNAs and radiotherapy}

Circulating miRNAs are utilized as important biomarkers of tumor-radiation response and toxicity. It has been underscored that the profiles of specific miRNAs in peripheral blood are significantly correlated with radiation type and dosimetry. ${ }^{59}$ They can be readily assessed by way of noninvasive blood testing. In murine serum, miRNA signatures are specifically changed following whole-body radiation. ${ }^{60}$ Circulating miRNA expression in human blood cells is induced upon irradiation, and can accurately distinguish pre- and postradiation situations. ${ }^{61}$ In lung cancer, circulating miR1246 in non-exosomeassociated form was actively altered by radiation exposure. This extracellular miR1246 could conntribute to the radiation-induced bystander effect. ${ }^{62}$ Moreover, it can enhance cell radioresistance and survival through directly suppressing DR5. ${ }^{63}$ In the circulation of NSCLC patients undergoing thoracic RT, miR29a and miR150 ae related to delivered radiation dose, and may eventually help predict the toxicity of RT. Moreover, the downregulation of miR29a was able to specifically reflect a profibrotic or adaptive situation in the human lung. ${ }^{64}$

\section{Exosomes and exosomal miRNAs in lung cancer}

Exosomal miRNAs serve as the pivotal model of circulating miRNAs existing stably in the extracellular environment. Exosomes are small membrane-derived vesicles (50-150 $\mathrm{nm}$ ) in eukaryotic fluids, and are released by multiple cell types. ${ }^{10}$ Overwhelming evidence indicates that exosomes originate from intraluminal endosomal vesicles inside multivesicular bodies. Fusion with the plasma membrane leads to extravasation of exosomes. ${ }^{65}$ Exosomes are pivotal in intracellular communication via transporting diverse molecular constitutuents. ${ }^{10}$ Exosomal cargoes are mainly small regulatory molecules, including miRNAs, mRNAs, and proteins (Figure 3). ${ }^{9}$ Among these, exosomal miRNAs display oncogenic or tumor-suppression activity in carcinogenesis, and potentially serve as biomarkers in clinical utility, having recently garnered enormous interest. ${ }^{66}$

In preclinical studies, how exosomal miRNAs mediate tumorigenesis and metastasis has been investigated 
Table I Roles of tissue-specific miRNAs in regulating lung cancer radiosensitivity

\begin{tabular}{|c|c|c|c|c|}
\hline miRNA & Target & Effect & Response & Reference \\
\hline $\operatorname{miR} 208 \mathrm{a}$ & p2I & Decreases cell apoptosis & Radioresistant & 11 \\
\hline $\operatorname{miR} 328-3 p$ & $\gamma \mathrm{H} 2 \mathrm{AX}$ & Inhibits survival, predicts poor outcome of NSCLC patients & Radiosensitive & 14 \\
\hline miR30a & ATFI & $\begin{array}{l}\text { Inhibits DNA-damage repair, blocks } G_{2} / M \text { arrest, and enhances radia- } \\
\text { tion-induced apoptosis }\end{array}$ & Radiosensitive & 15 \\
\hline miRI0I & $\begin{array}{l}\text { ATM and DNA- } \\
\text { PKcs }\end{array}$ & Inhibits DNA repair & Radiosensitive & 16 \\
\hline $\operatorname{miR} 1323$ & PRKDC & Enhances DNA-damage repair & Radioresistant & 17 \\
\hline $\operatorname{miR} 34 a$ & RAD5I & $\begin{array}{l}\text { Regulates HR, inhibits DSB repair, miR34a delivery (MRX34) plus RT } \\
\text { show therapeutic potential }\end{array}$ & Radiosensitive & 18 \\
\hline $\operatorname{miR} 25$ & BTG2 & Increases apoptosis & Radiosensitive & 22 \\
\hline $\operatorname{miR} 29 c$ & $\mathrm{Bcl} 2$ and $\mathrm{Mcl} \mathrm{I}$ & Increases apoptosis, longer relapse-free survival of patients & Radiosensitive & 24 \\
\hline $\operatorname{miR} 122$ & IGFIR & $\begin{array}{l}\text { Enhances DSBs, apoptosis, and anchorage-independent growth inhibi- } \\
\text { tion induced by IR }\end{array}$ & Radiosensitive & 25 \\
\hline miR5II & TRIB2 & Inhibits cell growth, increased apoptosis and triggers Bax activation & Radiosensitive & 26 \\
\hline $\operatorname{miR95}$ & SNXI & Decreases apoptosis & Radioresistant & 27 \\
\hline $\operatorname{miR} 124$ & STAT3 & Enhances radiation-induced apoptosis & Radiosensitive & 28 \\
\hline miR-198 & MET & Inhibits HGF-cMet signaling pathway, induces apoptosis & Radiosensitive & 29 \\
\hline miR2I & PDCD4 & $\begin{array}{l}\text { Decreases cell apoptosis, activates PI3K-Akt-mTOR pathway, shorter } \\
\text { median survival time }\end{array}$ & Radioresistant & 34,49 \\
\hline $\operatorname{miR} 210$ & - & Promotes hypoxic phenotype and DSB repair & Radioresistant & 35 \\
\hline $\operatorname{miR} \mid 48 b$ & ROCKI & Inhibits proliferation and EMT and promotes cell apoptosis & Radiosensitive & 38 \\
\hline $\operatorname{miR} 200 \mathrm{c}$ & - & $\begin{array}{l}\text { Activates EGFR-associated signaling, increases cell killing, and } \\
\text { regulatesEMT }\end{array}$ & Radiosensitive & 39 \\
\hline $\operatorname{miR} \mid 45$ & $\begin{array}{l}\text { OCT } 4 \text {, SOX2, and } \\
\text { Fascin I }\end{array}$ & suppressed the proliferation of CSCs and improved radioresistance & Radiosensitive & 41 \\
\hline $\operatorname{miR} 21 / 95$ & - & $\begin{array}{l}\text { Inhibit PTEN, SNXI, and SGPPI expression and elevates Akt } \\
\text { phosphorylation }\end{array}$ & Radioresistant & 42 \\
\hline $\operatorname{miR} I 8 a-5 p$ & ATM and HIFI $\alpha$ & Inhibits DNA repair; predicts radiosensitivity & Radiosensitive & 43 \\
\hline $\operatorname{miR} 373$ & TIMP2 & Regulates PI3K-Akt and Smad signaling pathways & Radioresistant & 45 \\
\hline $\operatorname{miR} 126$ & - & Promotes apoptosis & Radiosensitive & 46 \\
\hline $\operatorname{miR} 45 \mathrm{I}$ & - & Enhances apoptosis, activates PTEN & Radiosensitive & 47 \\
\hline miR99a & mTOR & Induces $G_{1}$ arrest and apoptosis & Radiosensitive & 48 \\
\hline $\operatorname{miR} 9$ & NRPI & $\begin{array}{l}\text { Regulates PI3K-Akt, MARK/RK, and NFKB pathways, and inhibits } \\
\text { apoptosis }\end{array}$ & Radioresistant & 50 \\
\hline miR9 and let-7 & - & Suppresses NFKBI & Radiosensitive & 51 \\
\hline $\mathrm{miR} / 5 \mathrm{a} / \mathrm{I} 6$ & TLRI & TLRI-NFKB pathway, increases apoptosis & Radiosensitive & 52 \\
\hline let-7a & - & Attenuates Kras expression & Radiosensitive & 53 \\
\hline $\operatorname{miR} I 55$ & TP53INPI & $\begin{array}{l}\text { Enhances IR-induced senescence and cell killing, regulates } p 53 \text { and p38 } \\
\text { MAPK pathways }\end{array}$ & Radiosensitive & 54 \\
\hline $\operatorname{miR} 214$ & FoxO4 & Inhibits apoptosis and senescence and regulates p38 MAPK & Radioresistant & 55 \\
\hline
\end{tabular}

Abbreviations: NSCLC, non-small-cell lung cancer; RT, radiotherapy; IR, ionizing radiation; PKcs, PK catalytic subunits; HR, homologous recombination; DSBs, doublestrand breaks; EMT, epithelial-mesenchymal transition.

(Table 2). Exosomal miR21, miR23a, and miR210 have been discovered to facilitate the development of tumor angiogenesis. ${ }^{67-69}$ In addition, exosomal miR23 was implicated in EMT, due to its significantly increased expression after mesenchymal transition. ${ }^{70}$ Exosomal miRNAs may also be potent regulators of chemoresistance. Evidence has demonstrated that exosomal miR96 elevates tumor resistance to cisplatin. ${ }^{71}$ By acting as tumor suppressors, exosome-derived miR512, miR373, and miR146a-5p are closely correlated with increased sensitivity to cisplatin. ${ }^{72,73}$

In clinical research, exosomal miRNAs can be considered important biomarkers for prediction, diagnosis, and prognosis by way of liquid biopsy. miR21 and miR155 have been suggested to predict recurrence and inimical outcomes in lung cancer $^{74}$ Exosomal miRNAs are 


\section{Exosome and exosomal miRNAs in lung cancer therapy}

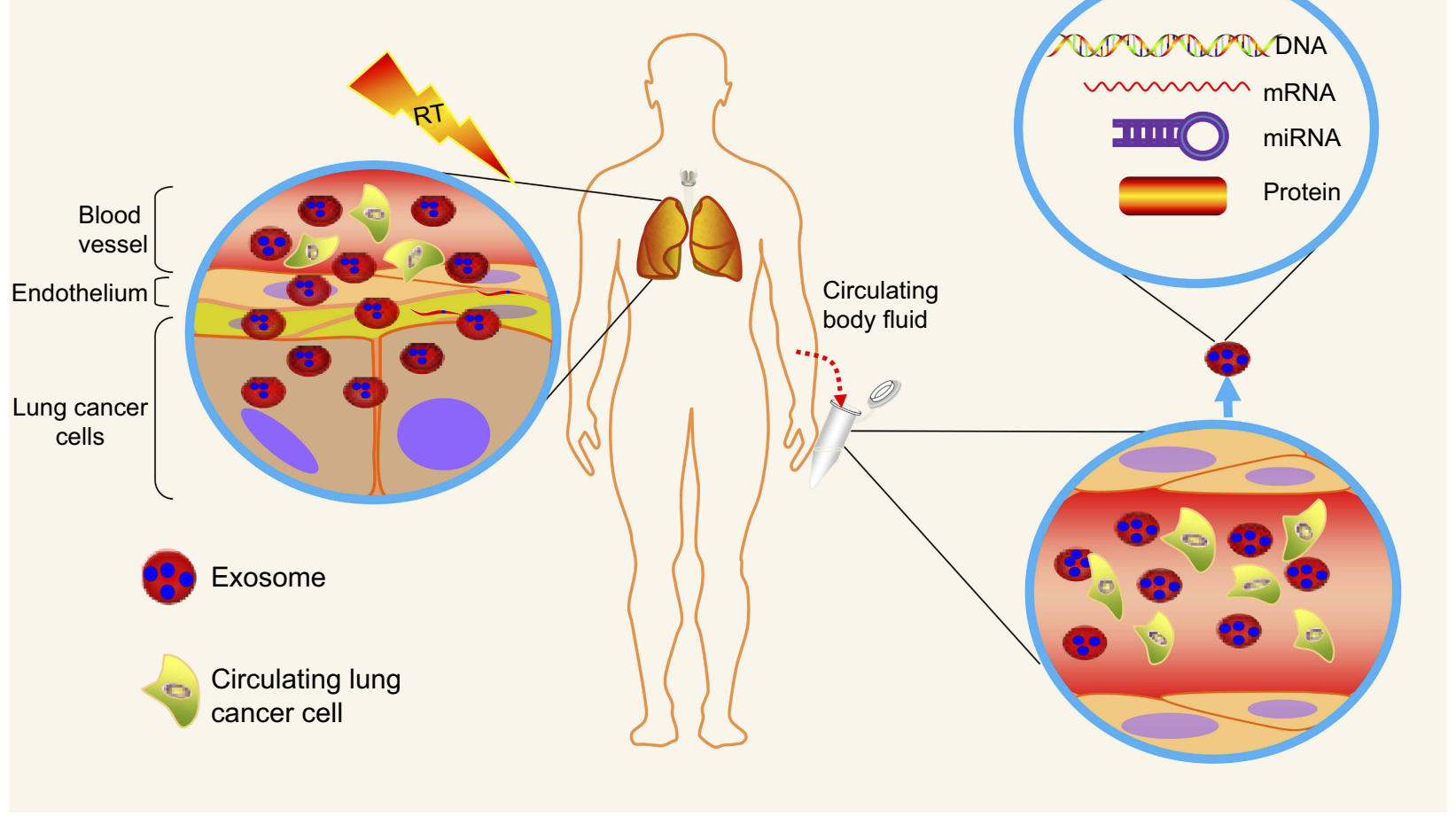

Figure 3 Exosomes and exosomal miRNAs in lung cancer therapy.

Notes: Exosomes are small membrane-derived vesicles in circulating body fluids that are released by multiple cell types, including tumor cells and normal cells. Exosomes specialize in intracellular communication, via transporting diverse molecular constitutes. Exosomal cargoes are mainly small regulatory molecules, including miRNAs, mRNAs, DNA, and proteins. Among them, exosomal miRNAs may be promising in regulating cellular radiosensitivity and monitoring radiotherapy effectiveness.

Table 2 Exosomal miRNAs in lung cancer

\begin{tabular}{|c|c|c|c|}
\hline Exosomal miRNA & Target & Effect & Ref. \\
\hline miR208a & P2I & $\begin{array}{l}\text { Promotes cell proliferation, induces radioresistance, activates Akt-mTOR } \\
\text { pathway }\end{array}$ & 11 \\
\hline miR2I & STAT3 & Promotes angiogenesis, transforms human bronchial epithelium & 61 \\
\hline miR23a & $\mathrm{PHDI} / 2$, ZOI protein & $\begin{array}{l}\text { Increases angiogenesis, vascular permeability, and cancer transendothelial } \\
\text { migration in hypoxic microenvironment }\end{array}$ & 62 \\
\hline miR210 & $\begin{array}{l}\text { FGFRLI, E2F3, VMPI, } \\
\text { RAD52, SDHD }\end{array}$ & $\begin{array}{l}\text { Increases angiogenesis by promoting tube-formation activity of umbilical } \\
\text { vein endothelial cell }\end{array}$ & 63 \\
\hline $\operatorname{miR} 96$ & LMO7 & Enhances cell viability, migration, and cisplatin resistance & 65 \\
\hline miR5I2-5p & TEAD4 & Reduces cisplatin-induced apoptosis, cell migration, and cell proliferation & 66 \\
\hline $\operatorname{miR} 373$ & RelA, PIK3CA & Reduces cisplatin-induced apoptosis and cell migration & 66 \\
\hline miRI46a-5p & Atg 12 & Decreases resistance to cisplatin and recurrence rates of patients & 67 \\
\hline $\operatorname{miR} 29 a-3 p, \operatorname{miR} I 50-5 p$ & - & Radiation dose-related & 75 \\
\hline
\end{tabular}

proficient at distinguishing lung cancer patients from healthy subjects. In contemporary research, investigators have isolated exosomes to evaluate expression profiles of 12 tumor-related miRNAs. Interestingly, average expression of exosomal miRNAs was upregulated dramatically in lung cancer cases compared with low expression in healthy individuals. ${ }^{75}$ Further, early diagnosis is necessary to decrease lung cancer mortality. Exosomal miRNA-seq uencing analysis was performed among 46 patients with stage I NSCLC and 42 controls. ${ }^{76}$ Using a four-marker panel (let7b-5p, let7e-5p, miR23a-3p, and miR486-5p), early-stage lung cancer was able to be detected in time, with satisfactory diagnostic performance (AUC 0.899, sensitivity $80.25 \%$, specificity $92.31 \%$ ). Meanwhile, the 
researchers clarified four adenocarcinoma-specific and three squamous carcinoma-specific exosomal miRNAs, potentially differentiating NSCLC subtypes. ${ }^{76}$

\section{Exosomes and exosomal miRNAs in radiotherapy}

Exosomes have become a horizon in RT, as cellular communication potentially affects cells exposed to irradiation. $^{77}$ Exosomes specialize in modification of radiobiological impact between irradiated cells and unirradiated cells, such as genomic instability and RT-induced bystander effects. In breast cancer, the interaction between stromal and tumor cells can make use of paracrine and juxtacrine pathways for driving radioresistance through RNA contained within exosomes. ${ }^{78}$ Furthermore, release of exosomes from irradiated glioblastoma cells is upregulated after radiation, effectively promoting the migration of receiving cells via altering molecular constitutents. In radiation-exposed head-and-neck cancer cells, exosomes trigger the enhancement of antiapoptotic factors and participate in DDR via transferal of cargoes. ${ }^{77}$ In addition, exosomal miR7 in irradiated brain cells can facilitate the induction of distant bystander autophagy in unrradiated lung tissue, by directly targeting $\mathrm{Bcl} 2$. $^{79}$ Following lung cancer RT, serum miR208a is induced and promotes radioresistance by affecting cell survival and intricate signaling transduction. More importantly, isolated exosomes have been found to contain RT-induced miR208a in patients with lung cancer and were also incorporated into recipient cells and subsequently modulated radioresistance. ${ }^{11}$ Other reports have discovered doserelated effects of exosomal miRNAs during thoracic radiation treatment. Researchers screened 752 exosome-derived miRNAs of locally advanced NSCLC patients, showing that increasing RT dosage reduced miR29a-3p and miR150-5p expression. ${ }^{64}$ To conclude, exosomes containing miRNAs can be considered a promising vehicle to regulate cellular radiosensitivity and a plausible tool to monitor RT efficacy.

\section{Clinical implications of miRNAs in personalized lung cancer radiotherapy Potential role of miRNAs as biomarkers for lung cancer-radiation response}

Several studies have reported that miRNAs show potential predictive utility in cancer treatment, including lung cancer RT. $^{44}$ By exploring whether miRNAs are suitable to be candidate markers for radiosensitivity, investigators classified NSCLC patients receiving postoperative RT into RTsensitive and RT-resistant groups. By screening miRNA profiles of two groups, they found 12 differentially expressed miRNAs in RT-sensitive patients, indicating better prognosis and lower cancer recurrence. ${ }^{46}$ Moreover, combining eleven circulating miRNAs with clinicopathological parameters generated a dose-response score that successfully identified patients with better survival and minimal treatment-related toxicity after high-dose $\mathrm{RT}^{8}$ miR148b downregulation was strongly correlated with low radiation response, advanced tumor stage, and distant metastasis. In particular, miR148b was an independent predictor of impoverished survival. ${ }^{80}$ Accelerated reproliferation in the late phase of RT usually leads to the failure of treatment. Serum let7 expression can reliably reflect tumor proliferation like Ki67 in fractional RT courses and help predict patient survival. ${ }^{81}$ Moreover, a multicenter controlled clinical trial has started, exploring plasma miRNAs that predict radiosensitivity in distinct fractionation regimes of palliative RT for advanced NSCLC (ClinicalTrials.gov NCT03074175).

In radiation research, miRNAs may act as biomarkers to minimize unexpected radiation-linked side effects. Serum miR29a-3p and miR150-5p show a dose-dependent expression pattern and might correlate with adverse events, such as pulmonary fibrosis. ${ }^{64}$ Likewise, overexpression of serum miR155 and miR221 predicts radiation esophagitis in NSCLC patients with radiochemotherapy. ${ }^{82}$ Actually, the assessment of miRNAs as a potential predictor of lung cancer $\mathrm{RT}$ is under way, and more well-designed trials are required to verify clinical utility.

\section{Targeting miRNAs in lung cancer radiotherapy}

With a deep understanding of miRNAs, quintessential miRNA-based therapies have come into the limelight of cancer management. Generally, therapeutics targeting miRNAs primarily comprise two approaches: miRNAinhibition therapy, which exploits miRNA antagonists or inhibitors to repress excessive activity of oncogenic miRNAs, and miRNA-replacement therapy, which uses miRNA mimics to compensate for defective function of tumor-suppressor miRNAs. ${ }^{83}$ The implementation of miRNA-based therapies is either by directly inhibiting miRNA sequences or by indirectly changing the coding sequence. For the latter, siRNAs and shRNAs encoded by 
vectors are always utilized. ${ }^{84}$ As a synthetic miR34 mimic delivered by liposomes, MRX34 is a pioneer miRNA therapeutic to enter the clinical arena. It has been evaluated in a phase I trial among patients with unresectable liver cancer or other advanced malignancies, including lung cancer (ClinicalTrials.gov NCT01829971).

Advances in miRNA-based therapies highlight an optimistic outlook for RT. Imaginative modes of targeting miRNAs in RT might contain the following steps: 1) before $\mathrm{RT}$, assessing the expression patterns of radiorelated miRNAs to predict radiation response and subsequently tailoring personalized RT plans based on individual radiosensitivity or tumor radioresistance; 2) during RT, altering the expression of specific miRNAs to accomplish the expected radiosensitizing effects, and analyzing liquid-biopsy samples, including exosomes, to capture dynamic characteristics of each patient in the treatment course; and 3) after RT, regularly tracking the expression of prognostic circulating miRNAs or further exosomal miRNAs to monitor RT efficacy and prevent cancer recurrence in timely fashion.

\section{Conclusion}

Lung cancer is the foremost deadly malignancy. RT is a significant treatment option for lung cancer. However, the existence of radioresistance largely limits the effectiveness of RT. There is a pressing need to decipher molecular mechanisms underlying radioresistance and elucidating novel therapeutic targets for individualized RT. Several studies have shown that tissue-specific miRNAs are extraordinary players in regulating cell radiosensitivity via intricate interplay with biological processes and radiationinduced signaling pathways. More importantly, exosomederived miRNAs have garnered immense attention in lung cancer treatment. Also, exosomal miRNAs are altered in response to radiation and hold considerable promise as diagnostic and therapeutic biomarkers. With advances in the understanding of miRNAs, miRNA-based therapy in conjunction with RT may be a promising strategy to tailor personalized treatment. However, to strengthen enthusiasm for targeting miRNAs in the clinic further, there remain some important challenges.

Firstly, molecular elucidation of radiobiological mechanisms and targets of miRNAs are fundamental for future implementation of miRNA-based therapies. We are also curious about the accurate roles of miRNAs in epigenetic mechanisms, such as DNA methylation and $N^{6}$ methyladenosine. Thorough understanding of the intricate interplay between miRNAs and radiation stress at different levels is urgently needed to broaden new horizons in the "mature" microRNA field. Additionally, the heterogeneity of the tumor microenvironment may largely influence miRNA signatures in radiation. Different patients with distinct genetic backgrounds have heterogeneous microenvironments and radiosensitivity. Moreover, tumor development during RT is dynamic, with different levels of miRNA expression. As such, future rigorous clinical studies should carefully consider tumormicroenvironment differences and keep constantly monitoring miRNAs during the whole period of RT. Thirdly, harnessing the potential of exosomes using noninvasive liquid biopsy is currently difficult. Despite considerable efforts in standardization techniques, there is no clear consensus on the best way to isolate and quantify exosomes. To date, exosomal miRNAs have displayed restricted clinical applicability in RT, because their potential function has not yet been appropriately studied in patient samples. More prospective investigations in patients receiving RT are vital to validate clinical utility. Last but not least, it appears that single miRNAs are imperfect to predict clinical outcomes consistently, whereas comprehensive analysis of miRNAs can improve their performance. miRNA-expression signatures may be combined with additional molecular, genetic, and clinicopathological information and noninvasive imaging approaches aiming to optimize personalized management.

In future, addressing these critical questions may bring great hope for radiation oncologists to tailor miRNA-based applications in the clinical arena, as well as lead us toward personalized lung cancer RT.

\section{Acknowledgments}

This work was funded by the National Natural Science Foundation of China (grant 81472799). The authors thank Dr Assain Ganiou, Department of Radiation and Medical Oncology, Zhongnan Hospital of Wuhan University for writing assistance.

\section{Disclosure}

The authors report no conflicts of interest in this work.

\section{References}

1. Siegel RL, Miller KD, Jemal A. Cancer statistics, 2018. CA Cancer J Clin. 2018;68(1):7-30. doi:10.3322/caac.21442

2. Travis WD, Brambilla E, Nicholson AG, et al. The 2015 world health organization classification of lung tumors: impact of genetic, clinical and radiologic advances since the 2004 classification. J Thorac Oncol. 2015;10(9):1243-1260. doi:10.1097/JTO.0000000000000630 
3. Lischalk JW, Woo SM, Kataria S, et al. Long-term outcomes of stereotactic body radiation therapy (SBRT) with fiducial tracking for inoperable stage I non-small cell lung cancer (NSCLC). J Radiat Oncol. 2016;5(4):379-387. doi:10.1007/s13566-016-0273-4

4. Willers H, Azzoli CG, Santivasi WL, Xia F. Basic mechanisms of therapeutic resistance to radiation and chemotherapy in lung cancer. Cancer J. 2013;19(3):200-207. doi:10.1097/PPO.0b013e318292e4e3

5. Rosenzweig KE, Gomez JE. Concurrent chemotherapy and radiation therapy for inoperable locally advanced non-small-cell lung cancer. J Clin Oncol. 2017;35(1):6-10. doi:10.1200/JCO.2016.69.9678

6. Bartel DP. MicroRNAs: genomics, biogenesis, mechanism, and function. Cell. 2004;116(2):281-297.

7. Czochor JR, Glazer PM. microRNAs in cancer cell response to ionizing radiation. Antioxid Redox Signal. 2014;21(2):293-312. doi:10.1089/ars.2013.5718

8. Sun Y, Hawkins PG, Bi N, et al. Serum microRNA signature predicts response to high-dose radiation therapy in locally advanced non-small cell lung cancer. Int J Radiat Oncol Biol Phys. 2018;100 (1):107-114. doi:10.1016/j.ijrobp.2017.08.039

9. Malla B, Zaugg K, Vassella E, Aebersold DM, Dal Pra A. Exosomes and exosomal MicroRNAs in prostate cancer radiation therapy. Int J Radiat Oncol Biol Phys. 2017;98(5):982-995. doi:10.1016/j. ijrobp.2017.03.031

10. Vanni I, Alama A, Grossi F, Dal Bello MG, Coco S. Exosomes: a new horizon in lung cancer. Drug Discov Today. 2017;22 (6):927-936. doi:10.1016/j.drudis.2017.03.004

11. Tang Y, Cui Y, Li Z, et al. Radiation-induced miR-208a increases the proliferation and radioresistance by targeting p21 in human lung cancer cells. J Exp Clin Cancer Res. 2016;35:7. doi:10.1186/ s13046-016-0444-6

12. Li L, Story M, Legerski RJ. Cellular responses to ionizing radiation damage. Int J Radiat Oncol Biol Phys. 2001;49(4):1157-1162.

13. Hu H, Gatti RA. MicroRNAs: new players in the DNA damage response. J Mol Cell Biol. 2011;3(3):151-158. doi:10.1093/jmcb/ mjq042

14. Ma W, Ma CN, Zhou NN, Li XD, Zhang YJ. Up- regulation of miR-328-3p sensitizes non-small cell lung cancer to radiotherapy. Sci Rep. 2016;6:31651. doi:10.1038/srep31651

15. Guo Y, Sun W, Gong T, et al. miR-30a radiosensitizes non-small cell lung cancer by targeting ATF1 that is involved in the phosphorylation of ATM. Oncol Rep. 2017;37(4):1980-1988. doi:10.3892/ or.2017.5448

16. Chen S, Wang H, Ng WL, Curran WJ, Wang Y. Radiosensitizing effects of ectopic miR-101 on non-small-cell lung cancer cells depend on the endogenous miR-101 level. Int J Radiat Oncol Biol Phys. 2011;81(5):1524-1529. doi:10.1016/j.ijrobp.2011.05.031

17. Li Y, Han W, Ni TT, et al. Knockdown of microRNA-1323 restores sensitivity to radiation by suppression of PRKDC activity in radiation-resistant lung cancer cells. Oncol Rep. 2015;33 (6):2821-2828. doi:10.3892/or.2015.3884

18. Cortez MA, Valdecanas D, Niknam S, et al. In vivo delivery of miR-34a sensitizes lung tumors to radiation through RAD51 regulation. Mol Ther Nucleic Acids. 2015;4:e270. doi:10.1038/ mtna.2015.47

19. Medema RH, Macurek L. Checkpoint control and cancer. Oncogene. 2012;31(21):2601-2613. doi:10.1038/onc.2011.451

20. Liu Q, Fu H, Sun F, et al. miR-16 family induces cell cycle arrest by regulating multiple cell cycle genes. Nucleic Acids Res. 2008;36 (16):5391-5404. doi:10.1093/nar/gkn522

21. Rahmanian N, Hosseinimehr SJ, Khalaj A. The paradox role of caspase cascade in ionizing radiation therapy. J Biomed Sci. 2016;23(1):88. doi:10.1186/s12929-016-0306-8

22. He Z, Liu Y, Xiao B, Qian X. miR-25 modulates NSCLC cell radio-sensitivity through directly inhibiting BTG2 expression. Biochem Biophys Res Commun. 2015;457(3):235-241. doi:10.1016/ j.bbrc.2014.12.094
23. Zhao L, Bode AM, Cao Y, Dong Z. Regulatory mechanisms and clinical perspectives of miRNA in tumor radiosensitivity. Carcinogenesis. 2012;33(11):2220-2227. doi:10.1093/carcin/bgs235

24. Arechaga-Ocampo E, Lopez-Camarillo C, Villegas-Sepulveda N, et al. Tumor suppressor miR-29c regulates radioresistance in lung cancer cells. Tumour Biol. 2017;39(3). 1010428317695010. doi: $10.1177 / 1010428317695010$

25. Ma D, Jia H, Qin M, et al. MiR-122 induces radiosensitization in non-small cell lung cancer cell line. Int J Mol Sci. 2015;16 (9):22137-22150. doi:10.3390/ijms160922137

26. Zhang HH, Pang M, Dong W, et al. miR-511 induces the apoptosis of radioresistant lung adenocarcinoma cells by triggering BAX. Oncol Rep. 2014;31(3):1473-1479. doi:10.3892/or.2014.2973

27. Ma W, Ma CN, Li XD, Zhang YJ. Examining the effect of gene reduction in miR-95 and enhanced radiosensitivity in non-small cell lung cancer. Cancer Gene Ther. 2016;23(2-3):66-71. doi:10.1038/cgt.2016.2

28. Wang M, Meng B, Liu Y, Yu J, Chen Q, Liu Y. MiR-124 inhibits growth and enhances radiation-induced apoptosis in non-small cell lung cancer by inhibiting STAT3. Cell Physiol Biochem. 2017;44 (5):2017-2028. doi:10.1159/000485907

29. Zhu L, Xue F, Xu X, et al. MicroRNA-198 inhibition of HGF/c-MET signaling pathway overcomes resistance to radiotherapy and induces apoptosis in human non-small-cell lung cancer. $J$ Cell Biochem. 2018;119(9):7873-7886.

30. Osinsky S, Zavelevich M, Vaupel P. Tumor hypoxia and malignant progression. Exp Oncol. 2009;31(2):80-86.

31. Marignol L, Coffey M, Lawler M, Hollywood D. Hypoxia in prostate cancer: a powerful shield against tumour destruction? Cancer Treat Rev. 2008;34(4):313-327. doi:10.1016/j.ctrv.2008.01.006

32. Harada $\mathrm{H}$, Itasaka $\mathrm{S}$, Zhu Y, et al. Treatment regimen determines whether an HIF-1 inhibitor enhances or inhibits the effect of radiation therapy. $\mathrm{Br}$ J Cancer. 2009;100(5):747-757. doi:10.1038/sj.bjc.6604939

33. Meijer TW, Kaanders JH, Span PN, Bussink J. Targeting hypoxia, HIF-1, and tumor glucose metabolism to improve radiotherapy efficacy. Clin Cancer Res. 2012;18(20):5585-5594. doi:10.1158/ 1078-0432.CCR-12-0858

34. Jiang S, Wang R, Yan H, Jin L, Dou X, Chen D. MicroRNA-21 modulates radiation resistance through upregulation of hypoxia-inducible factor-1alpha-promoted glycolysis in non-small cell lung cancer cells. Mol Med Rep. 2016;13(5):4101-4107. doi: $10.3892 / \mathrm{mmr} .2016 .5010$

35. Grosso S, Doyen J, Parks SK, et al. MiR-210 promotes a hypoxic phenotype and increases radioresistance in human lung cancer cell lines. Cell Death Dis. 2013;4:e544. doi:10.1038/cddis.2013.71

36. Marie-Egyptienne DT, Lohse I, Hill RP. Cancer stem cells, the epithelial to mesenchymal transition (EMT) and radioresistance: potential role of hypoxia. Cancer Lett. 2013;341(1):63-72. doi:10.1016/j.canlet.2012.11.019

37. Zhang Y, Zheng L, Huang J, et al. MiR-124 Radiosensitizes human colorectal cancer cells by targeting PRRX1. PLoS One. 2014;9(4): e93917. doi:10.1371/journal.pone.0093917

38. Luo H, Liang C. MicroRNA-148b inhibits proliferation and the epithelial-mesenchymal transition and increases radiosensitivity in non-small cell lung carcinomas by regulating ROCK1. Exp Ther Med. 2018;15(4):3609-3616. doi:10.3892/etm.2018.5845

39. Koo T, Cho BJ, Kim DH, et al. MicroRNA-200c increases radiosensitivity of human cancer cells with activated EGFR-associated signaling. Oncotarget. 2017;8(39):65457-65468. doi:10.18632/oncotarget.18924

40. Pajonk F, Vlashi E, McBride WH. Radiation resistance of cancer stem cells: the 4 R's of radiobiology revisited. Stem Cells. 2010;28 (4):639-648. doi:10.1002/stem.318

41. Chiou GY, Cherng JY, Hsu HS, et al. Cationic polyurethanes-short branch PEI-mediated delivery of Mir145 inhibited epithelial-mesenchymal transdifferentiation and cancer stem-like properties and in lung adenocarcinoma. $J$ Control Release. 2012;159(2):240-250. doi:10.1016/j.jconrel.2012.01.014 
42. Zhang J, Zhang $\mathrm{C}, \mathrm{Hu} \mathrm{L}$, et al. Abnormal Expression of miR-21 and miR-95 in Cancer Stem-Like Cells is Associated with Radioresistance of Lung Cancer. Cancer Invest. 2015;33 (5):165-171. doi:10.3109/07357907.2015.1019676

43. Chen X, Wu L, Li D, et al. Radiosensitizing effects of miR-18a-5p on lung cancer stem-like cells via downregulating both ATM and HIF-1alpha. Cancer Med. 2018;7(8):3834-3847. doi:10.1002/ cam4.1527

44. Korpela E, Vesprini D, Liu SK. MicroRNA in radiotherapy: miRage or miRador? $\mathrm{Br} J$ Cancer. 2015;112(5):777-782. doi:10.1038/ bjc. 2015.6

45. Guo Y, Jiang Y, Sang M, Xu C. Down-regulation of miR-373 increases the radiosensitivity of lung cancer cells by targeting TIMP2. Int J Biochem Cell Biol. 2018;99:203-210. doi:10.1016/j. biocel.2018.04.014

46. Wang XC, Du LQ, Tian LL, et al. Expression and function of miRNA in postoperative radiotherapy sensitive and resistant patients of non-small cell lung cancer. Lung Cancer. 2011;72(1):92-99. doi:10.1016/j.lungcan.2010.07.014

47. Tian F, Han Y, Yan X, et al. Upregulation of microrna-451 increases the sensitivity of A549 cells to radiotherapy through enhancement of apoptosis. Thorac Cancer. 2016;7(2):226-231. doi:10.1111/17597714.12318

48. Yin H, Ma J, Chen L, et al. MiR-99a enhances the radiation sensitivity of non-small cell lung cancer by targeting mTOR. Cell Physiol Biochem. 2018;46(2):471-481. doi:10.1159/000488615

49. Jiang Y, Chen X, Tian W, Yin X, Wang J, Yang H. The role of TGF-beta1-miR-21-ROS pathway in bystander responses induced by irradiated non-small-cell lung cancer cells. Br J Cancer. 2014;111 (4):772-780. doi:10.1038/bjc.2014.368

50. Xiong K, Shao LH, Zhang HQ, et al. MicroRNA-9 functions as a tumor suppressor and enhances radio-sensitivity in radio-resistant A549 cells by targeting neuropilin 1. Oncol Lett. 2018;15 (3):2863-2870. doi:10.3892/ol.2017.7705

51. Arora H, Qureshi R, Jin S, Park AK, Park WY. miR-9 and let-7g enhance the sensitivity to ionizing radiation by suppression of NFkappaB1. Exp Mol Med. 2011;43(5):298-304. doi:10.3858/ emm.2011.43.5.031

52. Lan F, Yue X, Ren G, et al. miR-15a/16 enhances radiation sensitivity of non-small cell lung cancer cells by targeting the TLR1/NF-kappaB signaling pathway. Int J Radiat Oncol Biol Phys. 2015;91(1):73-81. doi:10.1016/j.ijrobp.2014.09.021

53. Oh JS, Kim JJ, Byun JY, Kim IA. Lin28-let7 modulates radiosensitivity of human cancer cells with activation of K-Ras. Int $J$ Radiat Oncol Biol Phys. 2010;76(1):5-8. doi:10.1016/j.ijrobp.2009.08.028

54. Wang Y, Scheiber MN, Neumann C, Calin GA, Zhou D. MicroRNA regulation of ionizing radiation-induced premature senescence. Int J Radiat Oncol Biol Phys. 2011;81(3):839-848. doi:10.1016/j. ijrobp.2010.09.048

55. Salim H, Akbar NS, Zong D, et al. miRNA-214 modulates radiotherapy response of non-small cell lung cancer cells through regulation of p38MAPK, apoptosis and senescence. Br J Cancer. 2012;107 (8):1361-1373. doi:10.1038/bjc.2012.382

56. Weber JA, Baxter DH, Zhang S, et al. The microRNA spectrum in 12 body fluids. Clin Chem. 2010;56(11):1733-1741. doi:10.1373/ clinchem.2010.147405

57. Chen X, Liang H, Zhang J, Zen K, Zhang CY. Secreted microRNAs: a new form of intercellular communication. Trends Cell Biol. 2012;22 (3):125-132. doi:10.1016/j.tcb.2011.12.001

58. Valadi H, Ekstrom K, Bossios A, Sjostrand M, Lee JJ, Lotvall JO. Exosome-mediated transfer of mRNAs and microRNAs is a novel mechanism of genetic exchange between cells. Nat Cell Biol. 2007;9 (6):654-659. doi:10.1038/ncb1596

59. Pegtel DM, Cosmopoulos K, Thorley-Lawson DA, et al. Functional delivery of viral miRNAs via exosomes. Proc Natl Acad Sci U S A. 2010;107(14):6328-6333. doi:10.1073/pnas.0914843107
60. Templin T, Amundson SA, Brenner DJ, Smilenov LB. Whole mouse blood microRNA as biomarkers for exposure to gamma-rays and (56) Fe ion. Int $J$ Radiat Biol. 2011;87(7):653-662. doi:10.3109/ 09553002.2010.549537

61. Templin T, Paul S, Amundson SA, et al. Radiation-induced micro-RNA expression changes in peripheral blood cells of radiotherapy patients. Int J Radiat Oncol Biol Phys. 2011;80(2):549-557. doi:10.1016/j.ijrobp.2010.12.061

62. Prise KM, O'Sullivan JM. Radiation-induced bystander signalling in cancer therapy. Nat Rev Cancer. 2009;9(5):351-360. doi:10.1038/ $\operatorname{nrc} 2603$

63. Yuan D, Xu J, Wang J, et al. Extracellular miR-1246 promotes lung cancer cell proliferation and enhances radioresistance by directly targeting DR5. Oncotarget. 2016;7(22):32707-32722. doi:10.18632/ oncotarget.9017

64. Dinh TK, Fendler W, Chalubinska-Fendler J, et al. Circulating miR-29a and miR-150 correlate with delivered dose during thoracic radiation therapy for non-small cell lung cancer. Radiat Oncol. 2016;11:61. doi:10.1186/s13014-016-0636-4

65. Urbanelli L, Magini A, Buratta S, et al. Signaling pathways in exosomes biogenesis, secretion and fate. Genes (Basel). 2013;4 (2):152-170. doi:10.3390/genes4020152

66. Cui S, Cheng Z, Qin W, Jiang L. Exosomes as a liquid biopsy for lung cancer. Lung Cancer. 2018;116:46-54. doi:10.1016/j. lungcan.2017.12.012

67. Liu Y, Luo F, Wang B, et al. STAT3-regulated exosomal miR-21 promotes angiogenesis and is involved in neoplastic processes of transformed human bronchial epithelial cells. Cancer Lett. 2016;370 (1):125-135. doi:10.1016/j.canlet.2015.10.011

68. Hsu YL, Hung JY, Chang WA, et al. Hypoxic lung cancer-secreted exosomal miR-23a increased angiogenesis and vascular permeability by targeting prolyl hydroxylase and tight junction protein ZO-1. Oncogene. 2017;36(34):4929-4942. doi:10.1038/onc.2017.105

69. Cui H, Seubert B, Stahl E, et al. Tissue inhibitor of metalloproteinases-1 induces a pro-tumourigenic increase of miR-210 in lung adenocarcinoma cells and their exosomes. Oncogene. 2015;34(28):3640-3650. doi:10.1038/onc.2014.300

70. Kim J, Kim TY, Lee MS, Mun JY, Ihm C, Kim SA. Exosome cargo reflects TGF-beta1-mediated epithelial-to-mesenchymal transition (EMT) status in A549 human lung adenocarcinoma cells. Biochem Biophys Res Commun. 2016;478(2):643-648. doi:10.1016/j. bbrc.2016.07.124

71. Wu H, Zhou J, Mei S, et al. Circulating exosomal microRNA-96 promotes cell proliferation, migration and drug resistance by targeting LMO7. J Cell Mol Med. 2017;21(6):1228-1236. doi:10.1111/ jcmm. 13056

72. Adi Harel S, Bossel Ben-Moshe N, Aylon Y, et al. Reactivation of epigenetically silenced miR-512 and miR-373 sensitizes lung cancer cells to cisplatin and restricts tumor growth. Cell Death Differ. 2015;22(8):1328-1340. doi:10.1038/cdd.2014.221

73. Yuwen DL, Sheng BB, Liu J, Wenyu W, Shu YQ. MiR-146a-5p level in serum exosomes predicts therapeutic effect of cisplatin in non-small cell lung cancer. Eur Rev Med Pharmacol Sci. 2017;21 (11):2650-2658.

74. Yang M, Shen H, Qiu C, et al. High expression of miR-21 and miR-155 predicts recurrence and unfavourable survival in non-small cell lung cancer. Eur J Cancer. 2013;49(3):604-615. doi:10.1016/j. ejca.2012.09.031

75. Rabinowits G, Gercel-Taylor C, Day JM, Taylor DD, Kloecker GH. Exosomal microRNA: a diagnostic marker for lung cancer. Clin Lung Cancer. 2009;10(1):42-46. doi:10.3816/CLC.2009.n.006

76. Jin X, Chen Y, Chen H, et al. Evaluation of tumor-derived exosomal mirna as potential diagnostic biomarkers for early-stage non-small cell lung cancer using next-generation sequencing. Clin Cancer Res. 2017;23(17):5311-5319. doi:10.1158/10780432.CCR-17-0577 
77. Mutschelknaus L, Peters C, Winkler K, et al. Exosomes derived from squamous head and neck cancer promote cell survival after ionizing radiation. PLoS One. 2016;11(3):e0152213. doi:10.1371/journal. pone.0152213

78. Boelens $\mathrm{MC}, \mathrm{Wu} \mathrm{TJ}, \mathrm{Nabet} \mathrm{BY}$, et al. Exosome transfer from stromal to breast cancer cells regulates therapy resistance pathways. Cell. 2014;159(3):499-513. doi:10.1016/j.cell.2014.09. 051

79. Cai S, Shi GS, Cheng HY, et al. Exosomal miR-7 mediates bystander autophagy in lung after focal brain irradiation in mice. Int J Biol Sci. 2017;13(10):1287-1296. doi:10.7150/ijbs. 18890

80. Wang R, Ye F, Zhen Q, et al. MicroRNA-148b is a potential prognostic biomarker and predictor of response to radiotherapy in non-small-cell lung cancer. $J$ Physiol Biochem. 2016;72 (2):337-343. doi:10.1007/s13105-016-0485-5
81. Xie P, Li X, Tan X, Sun X, Wang C, Yu J. Sequential serum let-7 is a novel biomarker to predict accelerated reproliferation during fractional radiotherapy in lung cancer. Clin Lung Cancer. 2016;17(5): e95-e101. doi:10.1016/j.cllc.2016.03.010

82. Xu T, Liao Z, O'Reilly MS, et al. Serum inflammatory miRNAs predict radiation esophagitis in patients receiving definitive radiochemotherapy for non-small cell lung cancer. Radiother Oncol. 2014;113(3):379-384. doi:10.1016/j.radonc.2014.11.006

83. Florczuk M, Szpechcinski A, Chorostowska-Wynimko J. miRNAs as biomarkers and therapeutic targets in non-small cell lung cancer: current perspectives. Target Oncol. 2017;12(2):179-200. doi:10.1007/s11523-017-0478-5

84. Iqbal MA, Arora S, Prakasam G, Calin GA, Syed MA. MicroRNA in lung cancer: role, mechanisms, pathways and therapeutic relevance. Mol Aspects Med. 2018. doi:10.1016/j.mam.2018.07.003

\section{Publish your work in this journal}

Cancer Management and Research is an international, peer-reviewed open access journal focusing on cancer research and the optimal use of preventative and integrated treatment interventions to achieve improved outcomes, enhanced survival and quality of life for the cancer patient.
The manuscript management system is completely online and includes a very quick and fair peer-review system, which is all easy to use. Visit http://www.dovepress.com/testimonials.php to read real quotes from published authors. 